\section{Counterpoint: Wealth, Health Expenditure, and Cancer-Translating Research Into Efficient Policies}

\author{
Jad Chahoud, MD; Adele Semaan, MPH; and Alyssa Rieber, MD
}

Editor's note: This point and the previous commentary discuss the Chahoud et al study published in this issue of JNCCN (page 972). We welcome readers to contribute to the discussion by sending correspondence to jnccn@nccn.org or submitting it via www.editorialmanager.com/jnccn.

Sound science is the product of continuously evolving research. Our article in this issue of JNCCN (page 972) is one step toward answering a convoluted question about the associations between economic factors and cancer outcomes in the United States. Previous research on the determinants of cancer disparities has primarily focused on the local and regional levels. We adopted a different approach to assess these associations at the national level. We believe the 2 perspectives complement each other. For instance, understanding the interplay of wealth and expenditure as it impacts cancer outcomes in a city like Chicago is important to guide local policies and interventions. However, zooming out to capture the big picture, which has been understudied, is crucial to highlight disparities in cancer outcomes between different states, and this knowledge is necessary to objectively assess the role of national policies.

The goal of our study is not to (mis)guide policymakers; instead it is to highlight a problem of disparity and to fuel the discussion at the national level. The recommendation based on our findings is a call for "efficient policies needed to bend the health expenditure curve through emphasizing prevention, screening, and minimizing disparities in interstate cancer outcomes." Spending more does not necessarily result in better health outcomes; the United States has the highest health expenditure among the world's leading economies, but ranks 27th in life expectancy. We want to be clear that we are not recommending the "capping" of health spending. Instead, we are advocating for a better allocation of resources and for creating policies focused on "smart" spending. We believe that complementing financial resources with other community-based and low-cost preventive measures is critical, especially in prevalent cancers such as breast and colorectal cancers.

As noted in the conclusion of our paper, "Future research is needed to monitor the evolution of the highlighted interstate disparities in wealth, health expenditure, and cancer outcomes in light of the passage of the Patient Protection and Affordable Care Act in 2010." Our primary purpose is to lay the foundations for future studies in this field, and our end point is to facilitate the translation of this generated knowledge into more efficient policies aimed at improving universal cancer outcomes and eliminating disparities in the United States.
Jad Chahoud, MD

Jad Chahoud, MD, is from the Division of Cancer Medicine, The University of Texas MD Anderson Cancer Center, Houston, Texas.

Adele Semaan, MPH

Adele Semaan, MPH, is from the Division of Management Policy and Community Health at the University of Texas School of Public Health, Houston, Texas.
Alyssa Rieber, MD

Alyssa Rieber, MD, is from the Division of Cancer Medicine, Department of General Oncology, The University of Texas MD Anderson Cancer Center, Houston, Texas. 